\title{
MicroscopyPioneers
}

\section{Pioneers in Optics: Tycho Brahe}

From the website Molecular Expressions created by the late Michael Davidson and now maintained by Eric Clark, National Magnetic Field Laboratory,

Florida State University, Tallahassee, FL 32306

ecclark@magnet.fsu.edu

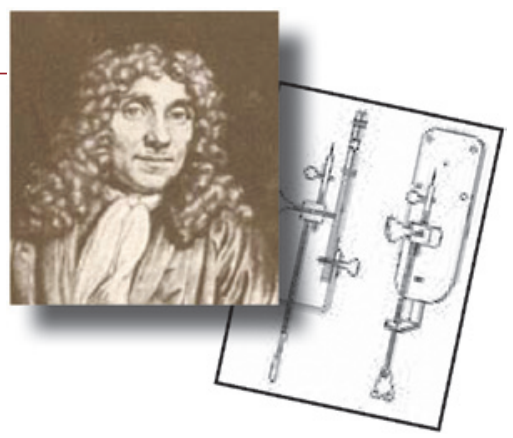

\section{Tycho Brahe}

(1546-1601)

Tycho Brahe was a Danish astronomer who made the most accurate observations possible without the aid of a telescope. His detailed astronomical findings led many to question the Aristotelian notion of a perfect and unchanging universe and laid the groundwork for future scientific breakthroughs.

Brahe was born the son of a nobleman on December 14, 1546, the surviving member of a pair of twin boys. He was raised, however, by a wealthy, childless uncle and became his heir. His rank afforded him an excellent education, and Brahe studied at the universities of Copenhagen and Leipzig, among others. Due to his uncle's desires Brahe entered school with the intention to prepare for a career in politics, but his own predilections led him to the field of astronomy. A discrepancy between the predicted and observed time of a partial eclipse of the sun perturbed Brahe, and he dedicated his life to making astronomy a more precise science in order to alleviate such inconsistencies. The death of his uncle in 1565 enabled

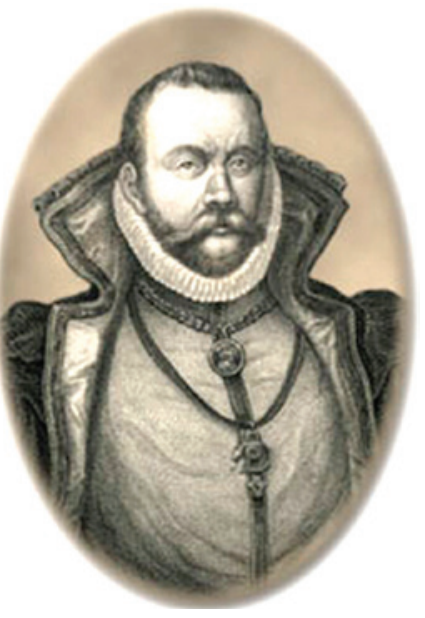
him to spend more time traveling and exploring his interests. Brahe accumulated scientific instruments, began composing his own star charts, and dabbled in alchemy.

Brahe returned to Denmark in 1570, where he lived with another uncle and set up his own observatory. From the homemade post, Brahe made a discovery that would make a profound impact on his life and on astronomy. On November 11, 1572, he observed what seemed to be a bright new star near Cassiopeia and studied it for the next 18 months. Brahe was surprised to find that the star seemed to be further away than the moon and that it intensified in brightness before eventually slowly fading out of view. The event was extremely significant because it would not have been possible if the Aristotelian conception of a harmonious and unchanging universe were correct.

After publishing a brief tract regarding his discovery in 1573, Brahe became an important figure in scientific circles. In 1574, he lectured on astronomy at the University of Copenhagen before embarking on a tour of Germany, on which he interacted with a number of other prominent astronomers. Fearing that Brahe would permanently relocate to Germany, in 1576, King Frederick II of Denmark offered to provide him with the money to develop a state-of-theart observatory on the island of Hven, which was located near Copenhagen. Brahe accepted the proposition, and construction soon began on the observatory that would be known as Uraniborg, or Castle of the Heavens. The facility, the first of its kind, attracted leading scholars from around the world.

In 1577, Brahe observed a bright comet, another phenomenon that did not correspond to the Aristotelian discipline. His measurements revealed that it, too, was more distant from the Earth than the moon, and could not, therefore, be an atmospheric occurrence as was suggested by Aristotle. Moreover, Brahe determined that the comet's path was elongate, rather than circular, which would mean that it would have to travel through the impenetrable spheres that were believed to carry the planets through the sky, a concept of Ptolemy. Brahe published his troubling findings that same year and also proposed a new theory regarding the system of the planets.

Unwilling to accept Copernicus's heliocentric theory, Brahe attempted to modify the Ptolemaic theory to coincide with his observations. In the Tychonic system, the Earth remained immobile, but the sun served as a secondary center. Mercury and Venus orbited around the sun, forming a small system that revolved around the Earth. Mars, Jupiter, and Saturn, Brahe suggested, orbited both the sun and the Earth, while the stars remained in a fixed sphere that made a full revolution each day. Although it was an interesting attempt at a compromise between two completely different viewpoints, the Tychonic system never garnered much support.

Upon the death of Frederick II, Brahe lost his financial backing and decided to relocate to Prague in 1597. There he again set up his instruments, many of which had been salvaged from his observatory at Hven, and obtained a new assistant, Johannes Kepler. When Brahe died following a brief illness in 1601, Kepler inherited all of Brahe's data and equipment. This act of bequeathal was yet another important scientific contribution made by Brahe because Kepler would use the information to develop his system of planetary motion. 


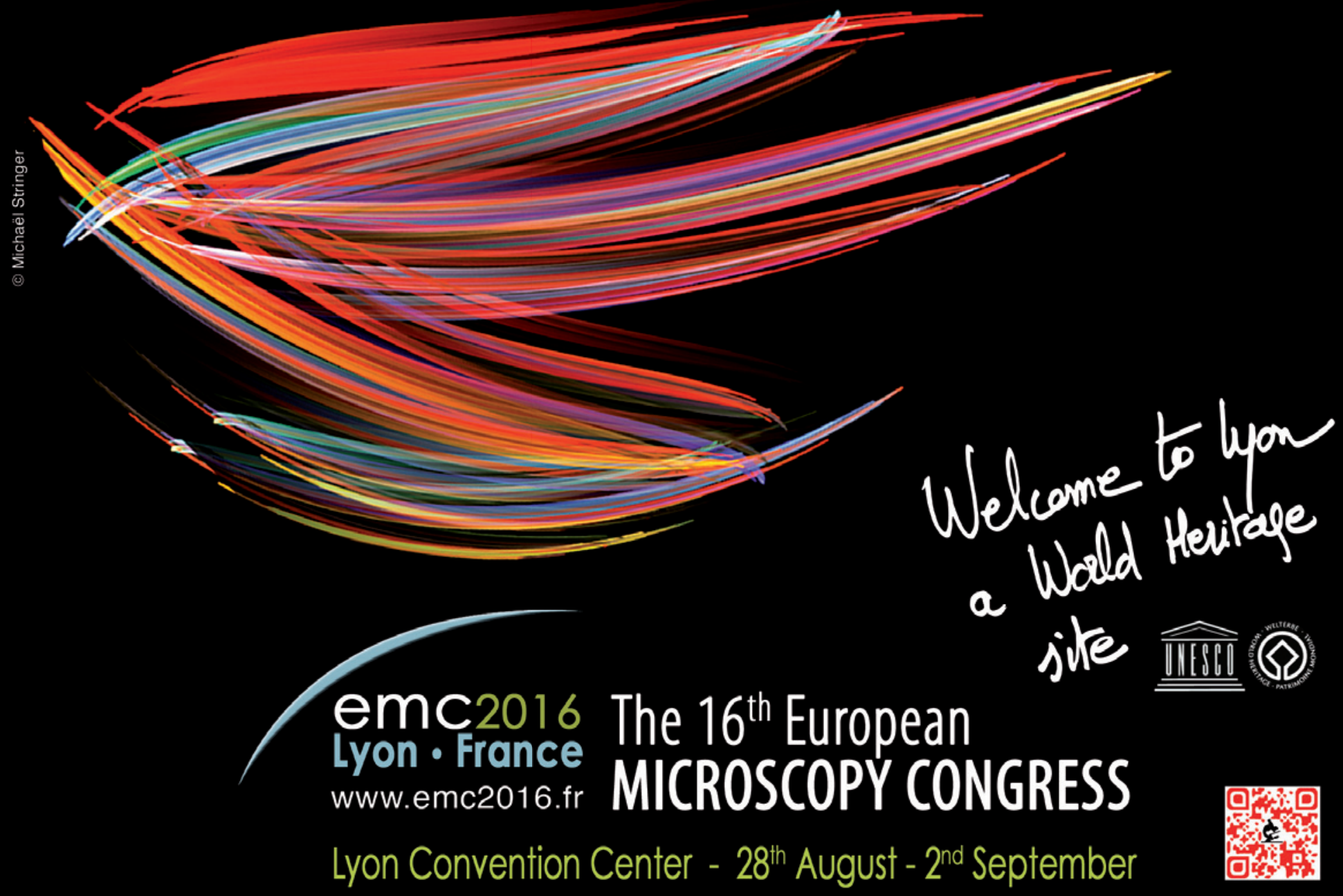

\section{PLENARY SPEAKERS}

Eric Betzig, USA

Nobel Prize 2014 Chemistry

"Super-resolved fluorescence microscopy"

\section{Jo Verbeek, Belgium}

Elektron vortex beams, link with optical techniques, applications to material science

\section{Bram Koster, Netherlands}

Biomedical imaging, correlative light and electron microscopy

\section{Nadine Peyriéras, France}

Morphogenesis, cell population dynamics, multiscale analysis, two-photon microscopy

\section{Frances Ross, USA}

Gas and liquid in situ / in operando (S)TEM applications to material science

\section{Hirofumi Yamada, Japan}

noise reduction in AFM for high resolution imaging or surfaces / interfaces of solids including solvation effects and electric-double layers

\section{SYMPOSIA}

800 POSTERS

3770 SQM EXHIBITION

AND MORE THAN 100 EXHIBITORS REPRESENTING ALL MICROSCOPIES

Organised by $\sqrt{\mu}$ and under the auspices of $M$ \& IFSM 